\title{
Dynamics of the Classical Two Dimensional XY Ferromagnet at Low Temperatures: a Memory Function Approach
}

\author{
S.A. Leonel \\ Departamento de Física, \\ Universidade Federal de Juiz de Fora, \\ 36036-330, Juiz de Fora, MG, Brazil \\ A.S.T. Pires \\ Departamento de Física, \\ Universidade Federal de Minas Gerais, \\ Caixa Postal 702, Belo Horizonte, 30161-970, MG, Brazil \\ Received 6 August 1998
}

\begin{abstract}
The spin dynamics of a two dimensional XY ferromagnet are reexamined at low temperatures in the framework of the Mori continued fraction formalism using the Gaussian approximation. In this formalism, the terms on denominators for the Laplace transform of the relaxation function $R(t)$ are related to the frequency moments $\left\langle\omega_{q}^{n}\right\rangle$ of the relaxation shape function $R(q, \omega)$. In the Gaussian approximation scheme, we truncate the continued fraction for $R(t)$ on the second stage. Adopting this approximation, we calculated up to the sixth moment. The moments are written in terms of the static spin correlation functions. In the estimation of the fourth and sixth moment at finite temperature, the four and sixspin correlation functions may be approximated by a sum of products of appropriate pair correlation functions (mode-mode decoupling). In this work we calculated the static spin correlation functions for the expressions to the fourth and sixth moments, needed in the study of the dynamics.
\end{abstract}

\section{Introduction}

It is well known that, in two dimensional (2D) planar magnetic spin systems, a topological phase transition referred to generally as Kosterlitz-Thouless(KT) transition is expected to occur at a temperature $[1,2]$ $T_{k T} \neq 0$. This transition is associated with the unbind of pair of vortices, which bind together to form vortex pairs of zero net helicity, as the temperature is raised above $T_{K T}$. The classical two-dimensional ferromagnetic models, the XY and the planar model, described by the same Hamiltonian

$$
H=-\frac{J}{2} \sum_{r, a}\left(S_{r}^{x} S_{r+a}^{x}+S_{r}^{y} S_{r+a}^{y}\right),
$$

where $\mathrm{J}$ is the exchange constant, have been studied in literature. In the XY model $\overrightarrow{S_{r}}$ (the spin at site $r$ ) is a three-dimensional classical rotor, while for the plane rotor model $\overrightarrow{S_{r}}$ is again a classical rotor but now it is constrained to two dimensions, having no z component, and therefore no dynamics. In this paper we will take $\left|\vec{S}_{r}\right|=S=1$. This produces no loss of generality when a classical system is under investigation, as the case of generic spin $\mathrm{S}$ is easily brought back by the rescaling of the exchange constant $J \rightarrow J S^{2}$.

For the XY model, as vortices and antivortices unbind they diffuse as a vortex gas $[3,4]$. The autocorrelations of the diffusing vortices give rise to a characteristic central (quasi elastic) peak in the dynamic structure factor $S(\vec{q}, \omega)$. Above $T_{K T}$ the dynamics of Hamilto- 
nian (1) has been extensively studied by Mertens et al. $[5,6]$.

In the low temperature regime the dynamics of (1) has been studied theoretically[7, 8] and numerically[4, 9] with different predictions for the nature of the dynamics structure factor $S(\vec{q}, \omega)$. The model was first analyzed by Villain[7] using the harmonic approximation. He found that $S(\vec{q}, \omega)$ had a spin wave peak of the form

$$
S(q, \omega) \approx\left|\omega-\omega_{q}\right|^{-1+\eta / 2}
$$

where $\eta$ is the critical exponent describing the decay of the static spin-spin correlation function and $\omega_{q}$ is the spin wave energy. Nelson and Fisher[10] treated the model in a fixed length hydrodynamic description. They obtained the following expression around the spin wave peak

$$
S(q, \omega) \approx \frac{1}{q^{3-\eta}} \frac{1}{\left|1-\omega^{2} / \omega_{q}^{2}\right|^{1-\eta}},
$$

where, for small $q, \omega_{q}=c q$ with $c=2 J$. Recently Menezes et al.[8] have performed a low temperature calculation using the projection operator technique. Besides a spin-wave peak, similar to that of Nelson and Fisher, they have found a logarithmically diverging central peak. In this paper we use the Mori-memory function formalism[11] in a Gaussian approximation[GA][12] to investigate the spin dynamics of Hamiltonian (1) at low temperatures. Our calculation remove the divergence obtained in pevius calculation. Recently Wang et al used Mori formalism and the decoupling of the four-spin correlation to investigate the spin $1 / 2$ Heisenberg antiferromagnet on a square lattice[13]. In Sect.II we calculate the static two-spin correlation functions, needed to study the dynamics. In Sect.III we present the calculation of $S(\vec{q}, \omega)$ and in Sect.IV our conclusions.

\section{Static Correlation Functions}

In order to calculate the static two-spin correlation functions for Hamiltonian (1) we start by using the polar representation for the spin at site $r$ :

$$
\vec{S}_{r}=\left(\sqrt{1-\left(S_{r}^{z}\right)^{2}} \cos \phi_{r}, \sqrt{1-\left(S_{r}^{z}\right)^{2}} \sin \phi_{r}, S_{r}^{z}\right)
$$

where we have taken $S=1$. Substituting(4) into (1), we find that keeping only terms of second order the Hamiltonian becomes

$$
H_{o}=\frac{J}{2} \sum_{r, a}\left[\frac{1}{2}\left(\phi_{r+a}-\phi_{r}\right)^{2}+\left(S_{r}^{z}\right)^{2}\right],
$$

It is easy to derive from the Fourier transform of (5) the following correlation functions

$$
<\left(S_{r}^{z}\right)^{2}>=T / 4 J
$$

$$
<\phi_{q} \phi_{-q}>=\frac{T}{4 J} \frac{1}{\left(1-\gamma_{q}\right)},
$$

where $\gamma_{q}=\frac{1}{2}\left(\cos q_{x}+\cos q_{y}\right)$ and $\mathrm{T}$ is the temperature.

Defining the in-plane symmetrized two-spin correlations function

$$
S_{r}^{\|}=\left\langle S_{o}^{x} S_{r}^{x}+S_{o}^{y} S_{r}^{y}>,\right.
$$

it is easily seen that [7]

$$
S_{r}^{\|}=\left(1-<\left(S_{o}^{z}\right)^{2}>\right) \exp [-(T / 4 J) g(r)]
$$

where

$g(r)=g(n, m)=\frac{1}{(2 \pi)^{2}} \int \frac{d \vec{K}\left(1-\cos K_{x} n \cos K_{y} m\right)}{1-\frac{1}{2}\left(\cos K_{x}+\cos K_{y}\right)}$.

and $r=\sqrt{n^{2}+m^{2}}$ in units of lattice spacing.

Calculating the integral numerically for the pair $(n, m)$ which will be used in static correlation function, we obtain

$$
\begin{array}{lll}
\mathrm{g}(0,0)=0.00 ; & \mathrm{g}(0,1)=1.00 ; & \mathrm{g}(1,1)=1.27 ; \\
\mathrm{g}(1,2)=1.54 ; & \mathrm{g}(0,2)=1.45 ; & \mathrm{g}(2,2)=1.70 ; \\
\mathrm{g}(0,3)=1.72 ; & \mathrm{g}(1,3)=1.76 ; & \mathrm{g}(0,4)=1.90 .
\end{array}
$$

Of course $<S_{0}^{x} S_{r}^{x}>=<S_{0}^{y} S_{r}^{y}>=S_{r}^{\|} / 2$.

The q-dependent correlation function is given by

$$
S_{q}^{\|}=\sum_{r} e^{i \vec{q} \cdot \vec{r}} S_{r}^{\|}=(1-T / 4 J) \tilde{S}(q),
$$

where

$$
\tilde{S}(q)=\sum_{r} e^{i \vec{q} \cdot \vec{r}} S(r),
$$

and

$$
S(r)=e^{-T g(r) / 4 J},
$$

which leads to 


$$
\tilde{S}(q)=\int_{0}^{\infty} \int_{-\pi}^{+\pi} e^{i q r \cos \theta} e^{-T g(r) / 4 J} r d r d \theta=2 \pi \int_{0}^{\infty} \mathcal{J}_{0}(q r) r e^{-T g(r) / 4 J} d r
$$

where $\mathcal{J}_{0}$ is the zeroth order Bessel function. At large distance $\mathrm{g}(\mathrm{r})$ can be approximated by [14]

$$
g(r)=\frac{2}{\pi} \ln \left(r / r_{0}\right),
$$

where $r_{0} \approx 0.2$, which leads to the well know result

$$
S(r)=\left(r_{0} / r\right)^{\eta}
$$

where $\eta=T / 2 \pi J$. In Fig. 1 we show $\mathrm{g}(\mathrm{r})$ given by Eq.15 with $m=0$, i.e, $g(n, 0)$, for simplicity and no loss of generality, calculated numerically as a function of $\ln (n)$. As we can see the approximation (15) works reasonably even for a few sites apart. Thus the powerlaw decay of the correlation is not strictly an asymptotic form for larger $r$, but works well also for small $r$. Therefore a more rapidly decaying portion, if present in addition to the $r^{-\eta}$-dependent term-, should not be important. This finding cost doubt in statement presented by Wiesler et al [2] that the presence of a more rapidly decaying portion could explain some discrepancies between theory and experimental data. Eq.(14), for small $q$, can be written as

$$
\tilde{S}(q)=\frac{2 \pi r_{0}^{\eta}}{q^{2-\eta}} \int_{0}^{\infty} \mathcal{J}_{0}(x) x^{1-\eta} d x
$$

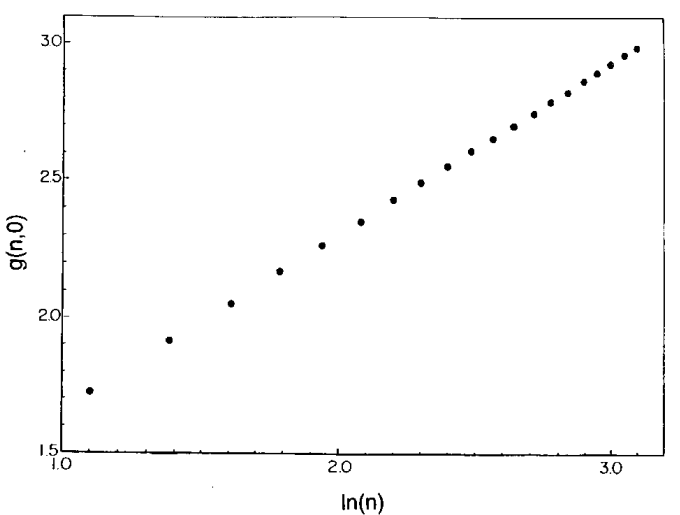

Figure 1. $g(n, 0)$ as a function of $\ln n$. The first point corresponds to $n=3$ The second to $n=4$ Tand so on.

Evaluating the integral we find

$$
\tilde{S}(\vec{q})=\frac{4\left(r_{0} / 2\right)^{\eta}}{q^{2-\eta}},{ }^{2}(1-\eta / 2) \sin (\pi \eta / 2)
$$

where, is the Gamma function.

\section{Dynamics}

In this Mori's formalism the Laplace transform of the relaxation function $R(t)=\left(S_{q}^{x}(t), S_{-q}^{x}\right) /\left(S_{q}^{x}, S_{-q}^{x}\right)$ is written as a continued fraction $[11,12]$ and given by

$$
\mathcal{R}(z)=\int_{0}^{\infty} R(t) e^{-z t} d t=\frac{1}{z+\delta_{1} /\left[z+\delta_{2} /\left(z+\delta_{3} /(z+\ldots)\right]\right.},
$$

where $\delta_{1}, \delta_{2}, \delta_{3}, \ldots$ are related to the frequency moments $\left\langle\omega_{q}^{n}\right\rangle$ of the relaxation shape function

$$
\tilde{R}(q, \omega)=\frac{1}{2 \pi} \int_{-\infty}^{\infty} e^{-i \omega t} R(t) d t=\frac{1}{\pi\left(<S_{q}^{x} S_{-q}^{x}>\right)} \operatorname{Re} \mathcal{R}(z=i \omega)
$$

through

$$
\delta_{2}=<\omega_{q}^{4}>/<\omega_{q}^{2}>-<\omega_{q}^{2}>
$$

$$
\delta_{1}=<\omega_{q}^{2}>,
$$




$$
\delta_{3}=\left(<\omega_{q}^{6}>/<\omega_{q}^{2}>-<\omega_{q}^{4}>^{2} /<\omega_{q}^{2}>^{2}\right) / \delta_{2} .
$$

The dynamics structure factor, defined by

$$
S(q, \omega)=\frac{1}{2 \pi} \int_{-\infty}^{\infty} d t e^{-i \omega t}<S_{q}^{x}(t) S_{-q}^{x}(0)>,
$$

is related to $\tilde{R}(q, \omega)$ by

$$
S(q, \omega)=\chi_{q} \omega\left(1-e^{-\beta \omega}\right)^{-1} \tilde{R}(q, \omega),
$$

where $\chi_{q}$ is the static susceptibility, which for a classical system is given by $\chi_{q}=\beta\left\langle S_{q}^{x} S_{-q}^{x}\right\rangle$, where $\beta=1 / T$ and we have taken the Boltzman constant equals to the unity. Within the GA scheme the continued fraction (19) is truncated to the second stage, yielding[12, 15]

$$
\tilde{R}(q, \omega)=\frac{1}{\pi} \frac{\delta_{1} \delta_{2} a_{2}(\omega)}{\left[\omega\left[\omega-\delta_{2} b_{2}(\omega)\right]-\delta_{1}\right]^{2}+\left[\omega \delta_{2} a_{2}(\omega)\right]^{2}}
$$

with

$$
\begin{gathered}
a_{2}=\frac{\pi}{\left(2 \delta_{3}\right)^{1 / 2}} \exp \left(-\omega^{2} / 2 \delta_{3}\right) \\
b_{2}=\frac{\exp \left(-\omega^{2} / 2 \delta_{3}\right)}{\left(\delta_{3} / 2\right)^{1 / 2}} \int_{0}^{s} e^{x^{2}} d x \\
s=\omega /\left(2 \delta_{3}\right)^{1 / 2}
\end{gathered}
$$

The integral in (28) was calculated numerically.

The moments for Hamiltonian (1) can be calculated using standard procedure $[12,17]$. The second moment has the exact relation with the two-spin correlation function

$$
<\omega_{q}^{2}>=4 J T<S_{0}^{x} S_{a}^{x}>/<S_{q}^{x} S_{-q}^{x}>
$$

The fourth moment is expressed in terms of static correlation functions of four spin operators

$$
<\omega_{q}^{4}>=T M_{4}(q) /<S_{q}^{x} S_{-q}^{x}>,
$$

with

$$
\begin{aligned}
M_{4}=-\sum_{q, p, t} & J_{q} J_{p} J_{t}\left(<S_{-p}^{x} S_{-q+t-k}^{x} S_{p+q+k}^{y}>\right. \\
& +<S_{-p}^{x} S_{p+q+t}^{x} S_{-q}^{y} S_{-t}^{y}> \\
& -2<S_{p+q+k}^{x} S_{-q+t-k}^{x} S_{-p}^{y} S_{-t}^{y}> \\
& -<S_{-q}^{y} S_{-t}^{y} S_{p+q+k}^{y} S_{-p+t-K}^{y}> \\
& +<S_{-p}^{y} S_{-q}^{y} S_{-t}^{y} S_{p+q+t}^{y}> \\
& +<S_{-q}^{y} S_{p+q+k}^{y} S_{t-k}^{z} S_{-p-t}^{z}> \\
& -<S_{-p}^{y} S_{-q}^{y} S_{t-k}^{z} S_{p+q+k-t}^{z}> \\
& +<S_{-t}^{y} S_{-p+t-k}^{y} S_{q+k}^{z} S_{p-q}^{z}> \\
& -<S_{q+k}^{z} S_{p-q}^{z} S_{-p-t}^{z} S_{t-k}^{z}> \\
& +<S_{-p}^{x} S_{p-q-t}^{x} S_{q+k}^{z} S_{t-k}^{z}> \\
& \left.+<S_{-p}^{x} S_{q+K_{T}}^{x} S_{p-q}^{z} S_{t-k}^{z}>\right), .
\end{aligned}
$$

where

$$
J_{q}=2 J\left(\cos q_{x}+\cos q_{y}\right) .
$$

The estimation of the fourth and sixth moment at finite temperature is complicated and an approximation is needed since there is no simple way in evaluating the four and six-spin correlation functions which are involved in the expression (32) and in a similar one for the sixth moment[16]. Let us, therefore, assume that the four and six-spin correlation functions may be approximated by a sum of products of appropriate pair correlation functions $[17,18,19]$ (mode-mode decoupling). Adopting this approximation we find

$$
\begin{aligned}
M_{4} \quad & =-2\left[-32<S_{0}^{x} S_{01}^{x}>\left(<\left(S_{0}^{x}\right)^{2}>+<S_{0}^{x} S_{02}^{x}>+2<S_{0}^{x} S_{11}^{x}>\right)\right. \\
& -8<S_{0}^{x} S_{01}^{x}><\left(S_{0}^{z}\right)^{2}>+\left(<S_{0}^{x} S_{01}^{x}>\left(18<S_{0}^{x} S_{12}^{x}>\right.\right. \\
& \left.\left.+3<S_{0}^{x} S_{03}^{x}>+43<S_{0}^{x} S_{01}^{x}>\right)+<\left(S_{0}^{z}\right)^{2}>^{2}\right)\left(\cos q_{x}+\cos q_{y}\right) \\
& -<\left(S_{0}^{z}\right)^{2}>\left(<\left(S_{0}^{x}\right)^{2}>+2<S_{0}^{x} S_{11}^{x}>\right. \\
& \left.\left.+<S_{0}^{x} S_{02}^{x}>\right)\left(\cos 2 q_{x}+\cos 2 q_{y}\right)\right],
\end{aligned}
$$


(where the following notation has been used: 10-nearest neighbor, 02 and 11 next nearest neighbor, and 12 and 03 third nearest neighbor).

At very low temperatures, we obtain the asymptotic expression:

$$
\begin{aligned}
& \delta_{1}=c^{2} q^{2}, \\
& \delta_{2}=8 t-c^{2} q^{2} t
\end{aligned}
$$

which leads to

$$
\begin{aligned}
& <\omega_{q}^{4}>\approx<\omega^{2}>^{2} \\
& <\omega_{q}^{6}>\approx<\omega^{2}>^{3},
\end{aligned}
$$

where $\mathrm{t}=\mathrm{T} / 4 \mathrm{~J}$, and $\mathrm{c}=2 \mathrm{~J}$. As $T \rightarrow 0, \delta_{2} \rightarrow 0$, nd $R(q, \omega)$ becomes a pair of delta functions at $\omega= \pm c q$, the spin wave frequency.

\section{Results}

The structure of $S(q, \omega)$ is ilustrated in Fig.2 and Fig.3 for $\mathrm{T} / \mathrm{J}=0.05$ and $\mathrm{T} / \mathrm{J}=0.10$ respectively and three values of the wave vector q(in reciprocal lattice units). For the largest wave vector the collective mode dominates $S(q, \omega)$ but for small $q$ there is considerable weight in $S(q, \omega)$ around $\omega=0$. The GA rounds off the divergence, found in former calculations [7, 8], of $S(q, \omega)$ at the spin wave frequency. The height of the resulting peaks will, however, diverge as $q$ and $\omega$ tend to zero. The divergence described in $[7,8]$ appears because an arbitrary number of "spin waves" contribute coherently to the dynamic structure factor. This happens became in those calculations the linewidt of a single magnon was not taken into account [20]. The behavior of the spin-wave peak is best described by Fig.4, where we show the peak position $\omega_{q}$ and the half-width at halfheight, as a function of $q$ for $\mathrm{T} / \mathrm{J}=0.01$, and Fig. 5 where we show $\omega_{q}$ and, as a function of $\mathrm{T}$ at $q=\pi / 10$.

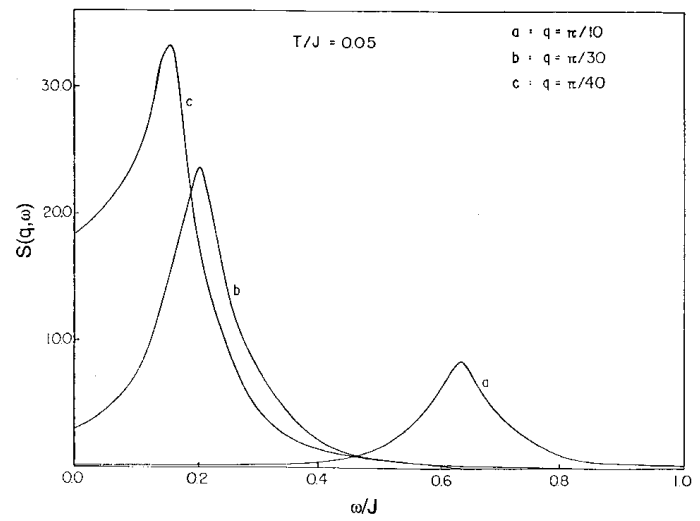

Figure 2. $S(q, \omega)$ is show for three wave vectors $\Gamma q=$ $\pi / 10, \pi / 30, \pi / 40$ and for $\mathrm{T} / \mathrm{J}=0.05$.

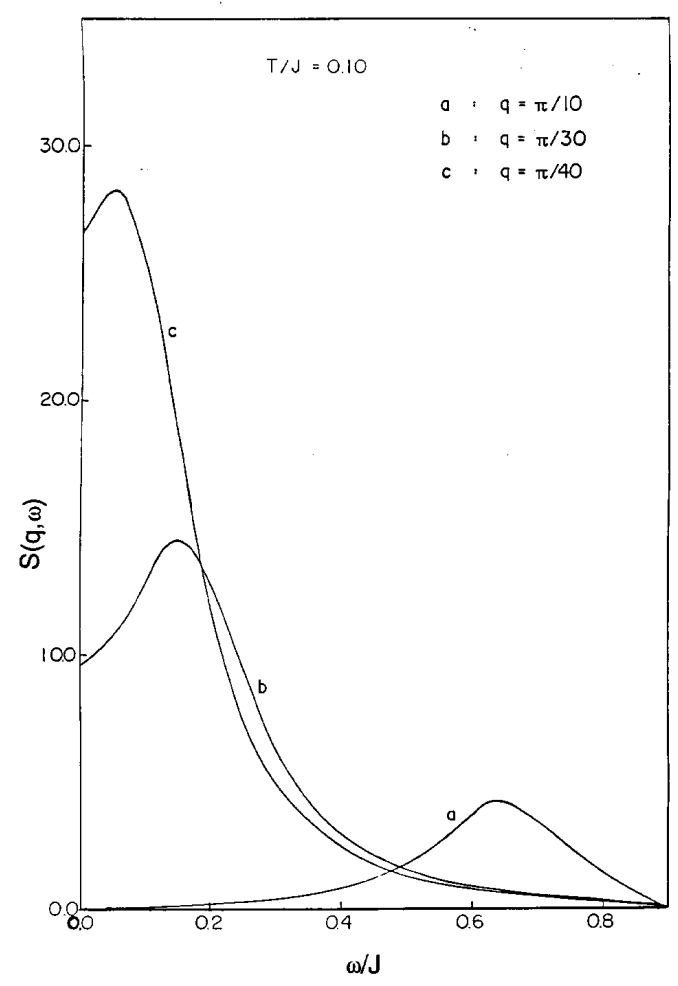

Figure 3. $S(q, \omega)$ is shown for three wave vectors $\Gamma q=$ $\pi / 10, \pi / 30, \pi / 40$ and for $\mathrm{T} / \mathrm{J}=0.10$.

As in similar calculations the use of the GA and 4-6spin correlation function decoupling can be questioned. However the theory developed here should provide, at least qualitatively, a description of the dynamics of the two dimensional XY model at low temperatures, and be a first step to a more elaborated calculation.

Up to now we do not know of any molecular dynamics simulation at low temperatures (to be compared with the theory here developed) but simulations at higher temperatures[9] shows a finite spin wave peak and the presence of a central peak. 


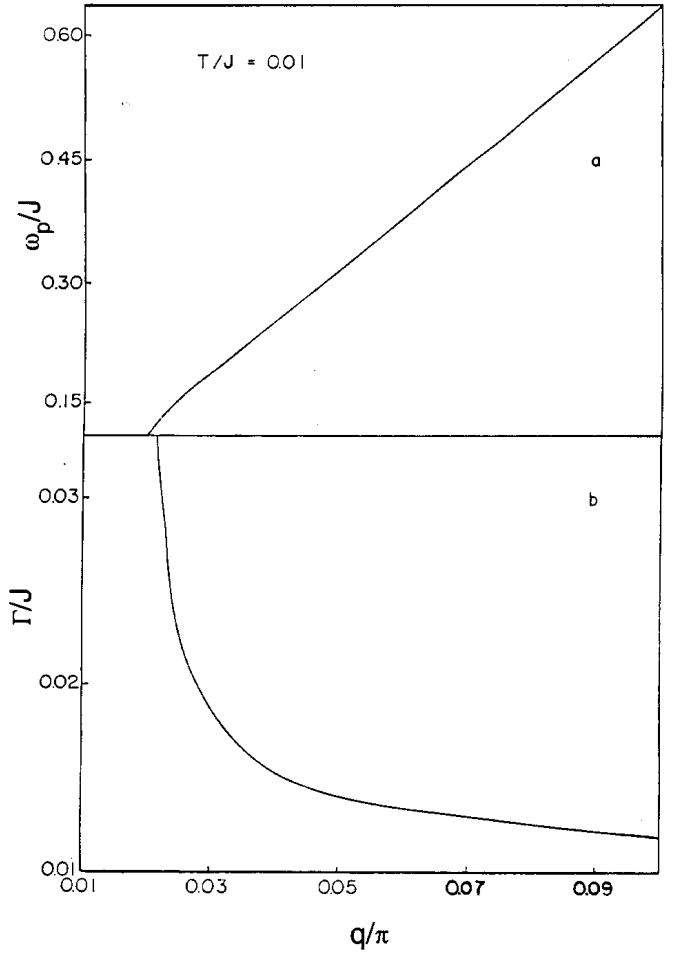

Figure 4. The spin-wave peak position $\omega_{q}(\mathrm{a})$ Tand the halfwidth at half-height $\Gamma(b)$ are show for $T / J=0.01$ and for wave vector in the range $0.01 \leq q / \pi \leq 0.1$.

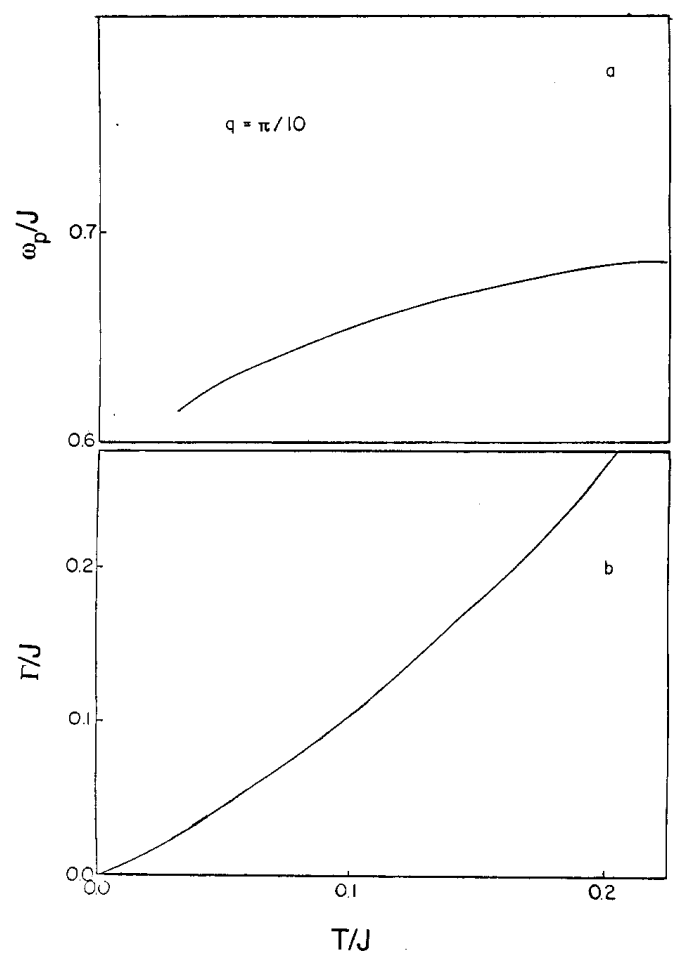

Figure 5. $\omega_{p}(a)$ and $\Gamma(b)$ are shown for $q=\pi / 10 \Gamma$ for temperatures in the range $0.0 \leq T / J \leq 0.02$.

\section{Acknowledgements}

We wish to thank W. Oliveira and F.Takakura for their valuable discussion on the calculation for the sixth moment.

This work was partially supported by Conselho Nacional de Desenvolvimento Cientıfico e Tecnolólogico(Brazil), and Capacitação e Aperfeiçoamento de Pessoal de Nível Superior(Brazil).

\section{References}

[1] J.M. KosterlitzГ D.J. ThoulessГ J. Phys. C6Г 1181 (1973).

[2] D.G. WieslerГH. ZabelГS.M. ShapiroTZ. Phys. В93Г 277 (1994) $\Gamma$ and references therein.

[3] D.L. HuberTPhys. Rev. B26Г3758 (1982).

[4] D.P. LandauT R.W. Gerling Г J. Magn. Mater. 104107Г843 (1992).

[5] F.G. MertensT A.R. BishopГ G.M. Wysin T C. Kawabata; Phys. Rev. Lett. 59Г 117 (1987); Phys. Rev. В39Г591 (1989).

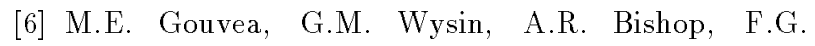
MertensГPhys. Rev. В39Г11840 (1989).

[7] J. Villain; J. Phys. (Paris) 35Г27 (1974).

[8] S.L. MenezesTA.S.T. PiresTM.E. GouveaTPhys. Rev. B47Г12280 (1993).

[9] H.G. Evertz and D.P. LandauTPhys. Rev. B54Г12302 (1995); J.F.R. Costa and B.V. CostaTPhys. Rev. B54 994 (1996).

[10] D.R. NelsonГ D.S. Fisher $\Gamma$ Phys. Rev. B16Г 4945 (1977).

[11] H. MoriГProg. Theor. Phys. 34Г399 (1965).

[12] A.S.T. PiresГHelv. Phys. Acta 61Г988 (1988).

[13] Y.J. Wang $\Gamma$ M.R. LiГ C.D. Gong $\Gamma$ Phys. Rev. B56Г 10982(1997).

[14] F. SpitzerT Principles of Random Walk. Princeton $\Gamma$ Van Nostrand (1964).

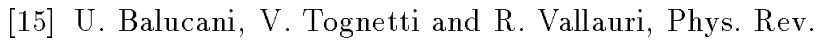
A19Г177 (1979).

[16] $\mathrm{Ph} . D$. thesis $\Gamma$ "Estudo da estática e dinâmica de sistemas de baixa dimensionalidade" $\Gamma$ Universidade Federal de Minas Gerais (1998).

[17] S.W. Lovesey[R.A. MeserveTPhys. Rev. Lett. 28Г614 (1971).

[18] S.W. LoveseyГR.A. MeserveГJ. Phys. C6Г79 (1973).

[19] A.P. Young TB.S. ShastryГJ. Phys. C15Г4547 (1982).

[20] M. SteinerT J. Villain and C.G. WindsorT Adv. Phys. $25 \Gamma 87$ (1976). 